\title{
Electromagnetic absorption mechanisms in metal nanospheres: Bulk and surface effects in radiofrequency-terahertz heating of nanoparticles
}

\author{
G. W. Hanson, ${ }^{1, a)}$ R. C. Monreal, ${ }^{2}$ and S. P. Apell ${ }^{3}$ \\ ${ }^{1}$ Department of Electrical Engineering, University of Wisconsin-Milwaukee 3200 N. Cramer St., Milwaukee, \\ Wisconsin 53211, USA \\ ${ }^{2}$ Departamento de Física Teórica de la Materia Condensada C05, Universidad Autónoma de Madrid, 28049 \\ Madrid Spain \\ ${ }^{3}$ Department of Applied Physics, Chalmers University of Technology, SE-41296 Göteborg, Sweden
}

(Received 12 April 2011; accepted 12 May 2011; published online 22 June 2011)

\begin{abstract}
We report on the absorption of electromagnetic radiation by metallic nanoparticles in the radio and far infrared frequency range, and subsequent heating of nanoparticle solutions. A recent series of papers has measured considerable radio frequency (RF) heating of gold nanoparticle solutions. In this work, we show that claims of RF heating by metallic nanoparticles are not supported by theory. We analyze several mechanisms by which nonmagnetic metallic nanoparticles can absorb low frequency radiation, including both classical and quantum effects. We conclude that none of these absorption mechanisms, nor any combination of them, can increase temperatures at the rates recently reported. A recent experiment supports this finding. (C) 2011 American Institute of Physics. [doi:10.1063/1.3600222]
\end{abstract}

\section{INTRODUCTION}

Absorption of electromagnetic radiation by metallic nanoparticles in the visible and infrared parts of the energy spectrum has attracted renewed interest due to potential applications in medicine and biology. ${ }^{1}$ The irradiated particles can absorb energy, one channel being the excitation of surface plasmons, and this energy can also be transferred to their surrounding medium leading to a variety of uses, such as markers or in ablation of cancer tumors. Individual nanoparticles must be subject to an intense electric field to heat substantially; e.g., in Ref. 2 individual gold nanoparticles heated by several hundred degrees when the incident laser intensity was $I \sim 1 \mathrm{GW} / \mathrm{m}^{2}$, yet individual nanoparticles are projected to heat far less than a degree for intensities on the order of $10^{4} \mathrm{~W} / \mathrm{m}^{2}$ (Ref. 3). However, even for the latter case, large collections of nanoparticles can generate significant heating (tens to hundreds of degrees) over macroscopic volumes. ${ }^{3}$ Alternately, because of the relatively low penetration depth of radiation at infrared (IR) and visible frequencies, radio frequency (RF) energy has been suggested for biomedical applications because it penetrates easily in the human body, thus reaching important internal organs. A series of papers ${ }^{4-12}$ has considered RF heating of gold nanoparticles, showing significant heating. However, a recent experiment $^{13}$ contradicts this, and finds that gold nanoparticles at RF do not heat. It was concluded that at RF the observed heating was due to Joule heating of the ionic stock solution which housed the nanoparticles. The purpose of this paper is to show that absorption of $\mathrm{MHz}$ through low $\mathrm{THz}$ radiation by individual, noninteracting, spherical metallic nanoparticles is negligible and produces no substantial heating and, further, that the heating is so insignificant that even very large collections of nanoparticles cannot lead to heating of the suspension, supporting the conclusions of Ref. 13. To

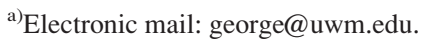

this end, we analyze several mechanisms by which nonmagnetic metallic nanoparticles can absorb low frequency radiation, namely, (i) the classical prediction of Mie theory for the absorption of electromagnetic energy by small spheres, including the possibility that the spheres are coated by an absorbing material, and (ii) electronic absorption occurring at the surface of the spheres due to electron spillout, surface roughness, and effects of surface phonons. We conclude that none of these absorption mechanisms, nor any combination of them, can increase temperatures at the rates recently reported in Refs. 4-12 at $\mathrm{MHz}$ frequencies. We note that the same conclusions can be made for frequencies at least through the low $\mathrm{THz}$ regime.

\section{CLASSICAL THEORY}

For a general material sphere of radius $R$ immersed in an infinite host medium having real-valued relative permittivity $\varepsilon_{h}$, the extinction and scattering cross sections are ${ }^{14}$

$$
\begin{gathered}
C_{e x t}=\frac{2 \pi}{k_{h}^{2}} \sum_{n=1}^{\infty}(2 n+1) \operatorname{Re}\left(a_{n}+b_{n}\right), \\
C_{s c a}=\frac{2 \pi}{k_{h}^{2}} \sum_{n=1}^{\infty}(2 n+1)\left(\left|a_{n}\right|^{2}+\left|b_{n}\right|^{2}\right),
\end{gathered}
$$

where $k_{h}=(\omega / c) \sqrt{\varepsilon_{h}}, \omega$ is the angular frequency of the EM field, $c$ is the speed of light in vacuum and $a_{n}$ and $b_{n}$ are the Mie coefficients representing electric and magnetic multipoles. The absorption cross section is $C_{a}=C_{e x t}-C_{s c a}$. For very small material spheres $\left(k_{h} R \ll 1\right)$ we can ignore scattering compared to extinction.

For a small nonmagnetic sphere, the leading terms of the Mie coefficients $\left(e^{-i \omega t}\right.$ dependence) are

$$
a_{1}=-i \frac{2\left(k_{h} R\right)^{3}}{3} \frac{\varepsilon-\varepsilon_{h}}{\varepsilon+2 \varepsilon_{h}}
$$




$$
b_{1}=-i \frac{\left(k_{h} R\right)^{5}}{45}\left(\frac{\varepsilon-\varepsilon_{h}}{\varepsilon_{h}}\right) \text {. }
$$

The corresponding absorption cross section of the sphere is then

$$
C_{a} \simeq \frac{6 \pi}{k_{h}^{2}} \operatorname{Re}\left(a_{1}+b_{1}\right)=k_{h} \operatorname{Im}\left(\alpha^{e}+\alpha^{m}\right) \equiv C_{a}^{e}+C_{a}^{m}
$$

where $\alpha^{e}$ is the electric dipole polarizability and $\alpha^{m}$ is the magnetic dipole polarizability (associated with eddy currents). Since we are studying good conductors at low frequencies, $b_{1}$ can dominate $a_{1}$ despite the extra factor of $\left(k_{h} R\right)^{2}$, except for the very smallest spheres.

We will assume $\varepsilon(\omega)$ to be given by the Drude formula

$$
\varepsilon(\omega)=1-\frac{\omega_{p}^{2}}{\omega\left(\omega+i / \tau_{0}\right)},
$$

where $\tau_{0}$ is the bulk relaxation time and $\omega_{p}$ is an effective plasma frequency. This is a good fit for noble metals at low frequencies.

\section{A. Small coated sphere}

In many instances nanoparticles may have a coating, such as an oxide layer, sodium citrate used in gold nanoparticle fabrication, or a protein for selective binding to a cell. For a sphere having radius $R$ and relative permittivity $\varepsilon$, with a coating having thickness $d$ and relative permittivity $\varepsilon_{\text {coat }}$, embedded in a host medium having real-valued relative permittivity $\varepsilon_{h}$, the electric dipole polarizability is ${ }^{14}$

$$
\begin{aligned}
\alpha^{e, c s}= & 3 V_{\text {cnp }} \\
& \times \frac{\left(\varepsilon_{\text {coat }}-\varepsilon_{h}\right)\left(\varepsilon+2 \varepsilon_{\text {coat }}\right)+r_{c}\left(\varepsilon-\varepsilon_{\text {coat }}\right)\left(\varepsilon_{h}+2 \varepsilon_{\text {coat }}\right)}{\left(\varepsilon_{\text {coat }}+2 \varepsilon_{h}\right)\left(\varepsilon+2 \varepsilon_{\text {coat }}\right)+2 r_{c}\left(\varepsilon_{\text {coat }}-\varepsilon_{h}\right)\left(\varepsilon-\varepsilon_{\text {coat }}\right)},
\end{aligned}
$$

where $V_{c n p}$ is the volume of the coated particle and $r_{c} \equiv[R /(R+d)]^{3}$. The magnetic polarizability is

$$
\alpha^{m, c s}=\frac{V_{c n p}}{10}\left(k_{h} R\right)^{2}\left(\frac{\varepsilon-\varepsilon_{\text {coat }}}{\varepsilon_{h}}\right)\left\{1+\frac{\left[k_{h}(R+d)\right]^{2}}{3}\right\}
$$

which is very weakly dependent on coating thickness. Even if the core is a good metal, the presence of an absorptive coating causes the electric dipole contribution to be very large compared to the magnetic dipole contribution through most of the $\mathrm{GHz}$ range.

The presence of even a very thin, slightly absorbing coating can have a profound influence on the absorbing properties of a particle. ${ }^{15-17}$ For example, let the host medium be an insulator with $\varepsilon_{h}=80$, and assume an $R=20 \mathrm{~nm}$ gold sphere $\left(\omega_{p} \simeq 1.37 \times 10^{16} \mathrm{~s}^{-1}, \tau_{0} \simeq 2 \times 10^{-14} \mathrm{~s}\right.$, which corresponds to a dc conductivity of $\sigma=3.3 \times 10^{7} \mathrm{~S} / \mathrm{m}$ ). The absorption cross section using $\tau_{0}$, normalized by the cross-sectional area $A=\pi R^{2}$, is shown in Fig. 1 (solid line) from $1 \mathrm{MHz}$ to 1 THz. The addition of a $2 \mathrm{~nm}$ thick coating with $\operatorname{Re}\left(\varepsilon_{\text {coat }}\right)=4$ and $\sigma_{\text {coat }}=0.01 \mathrm{~S} / \mathrm{m}\left[\operatorname{Im}\left(\varepsilon_{\text {coat }}\right)=13.8\right.$ at $\left.13 \mathrm{MHz}\right]$ increases

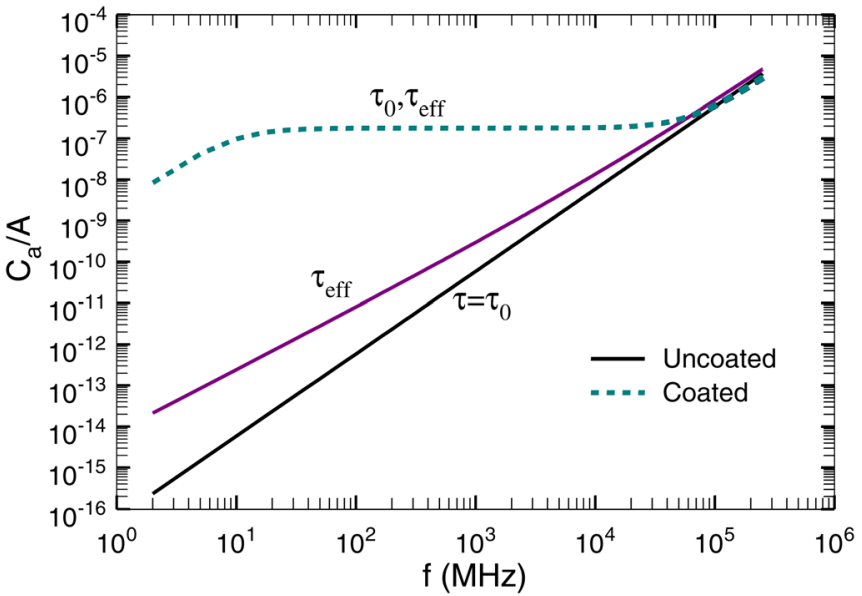

FIG. 1. (Color online) Absorption cross section normalized by cross-sectional area $A=\pi R^{2}$ for a $R=20 \mathrm{~nm}$ gold sphere immersed in an insulating host medium having $\varepsilon_{h}=80$ (solid line, $\tau=\tau_{0}$ ). The result for a coated sphere having $d=2 \mathrm{~nm}, R e \varepsilon_{\text {coat }}=4$, and $\sigma_{\text {coat }}=0.01 \mathrm{~S} / \mathrm{m}$ is also shown [dashed line, $A=\pi(R+d)^{2}$ ]. The curves for $\tau=\tau_{\text {eff }}$ account for nonlocal effects as discussed in Sec. III.

the absorption cross section by six orders of magnitude compared to the uncoated sphere at a typical RF frequency of 13 $\mathrm{MHz}$, as seen in Fig. 1 (dashed line - in this case the absorption cross section is normalized by $A=\pi(R+d)^{2} \simeq \pi R^{2}$ ). Also shown in Fig. 1 are the corresponding values computed using an effective relaxation time $\tau_{\text {eff }}$ accounting for nonlocal effects, described in Sec. III. Note that the host medium having a constant value of $\varepsilon_{h}=80$ can be considered to be pure water up until approximately $1 \mathrm{GHz}$, after which water dispersion and absorption become important; these effects will be considered later.

In Fig. 1, we have assumed a mildly conducting coating; Eq. (40) in Ref. 17 gives the coating conductivity that maximizes absorption, and the value $\sigma=0.01 \mathrm{~S} / \mathrm{m}$ maximizes absorption in a wide region of frequencies starting near 10 MHz. Many practical coatings may be expected to have conductivities on the order of $0.001-1 \mathrm{~S} / \mathrm{m}$; one estimate ${ }^{18}$ of the conductivity of sodium citrate buffer solutions is $0.25 \mathrm{~S} / \mathrm{m}$.

However, as we will show below, even for the coated sphere the absorption cross section is approximately eight orders of magnitude too small to account for the heating observed in the aforementioned papers (e.g., Ref. 7), even with a coating that maximizes absorption, and, in fact, this is true through the low $\mathrm{THz}$ regime. A model has been proposed in Ref. 7 to explain the observed RF heating, but this model cannot be correct because it computes absorption by multiplying conductivity inside the particle by the external (applied) electric field value, rather than using the electric field inside the particle (which, due to the high conductivity of the particle, is smaller by many orders of magnitude compared to the applied field).

The inability to explain significant RF absorption using the classical model leads to consideration of other factors, such as nonlocal surface effects. These have earlier been shown to increase the absorption cross section of small spheres in the visible or IR part of the EM spectrum. ${ }^{19,20}$ Their role at $\mathrm{RF}$ through $\mathrm{THz}$ frequencies is investigated next. 


\section{NONLOCAL ELECTRONIC SURFACE EFFECTS - ELECTRON SPILLOUT AND SURFACE ROUGHNESS}

It has been known for a long time ${ }^{20,21}$ that nonlocal surface effects on the electromagnetic response of small spheres (a few nanometers in size) can be described by means of two lengths, $d_{r}$ and $d_{\theta}$, defined as

$$
d_{r}(\omega)=\frac{1}{\frac{\varepsilon}{\varepsilon_{h}}-1} \int_{0}^{\infty} d r \frac{E_{r}(r)-E_{r}^{c l}(r)}{E_{r}^{c l}(R)}
$$

and

$$
d_{\theta}(\omega)=\int_{0}^{\infty} d r \frac{r}{R} \frac{D_{\theta}(r)-D_{\theta}^{c l}(r)}{E_{\theta}^{c l}(R)} .
$$

In Eqs. (8) and (9), $E_{r}$ is the radial component of the electric field and $D_{\theta}$ is the component of the electric displacement tangential to the surface for a realistic (non-abrupt) surface model; $E_{r}^{c l}$ and $D_{\theta}^{c l}$ are their classical counterparts for an abrupt particle boundary. Since the actual EM fields are continuous functions at the metal/medium interface, contrarily to the classical theory, $d_{r}$ and $d_{\theta}$ measure the response of a realistic metal nanoparticle surface to external radiation. In particular, the imaginary parts of both functions are related to absorption of energy by surface modifications of the classical bulk response. While $\operatorname{Im} d_{r}$ describes absorption by electronhole pairs taking into account the actual electron density profile at the surface - electron spillout, $\operatorname{Im} d_{\theta}$ describes absorption by diffuse electron surface scattering. If we assume a classical abrupt interface then both $d_{r}=0$ and $d_{\theta}=0$ by their definition in Eqs. (8) and (9), respectively. For realistic surfaces, $d_{r, \theta}$ are typically in the range of $0.1 \mathrm{~nm}$.

These surface effects can be appropriately taken into account by defining an effective dielectric function for a metal nanoparticle as ${ }^{20,22}$

$$
\varepsilon_{e f f}(\omega)=\varepsilon \frac{1+\frac{2}{\varepsilon} \frac{d_{\theta}}{R}}{1+\left(\frac{\varepsilon}{\varepsilon_{h}}-1\right) \frac{d_{r}}{R}} .
$$

Then, the electric dipolar contribution to the absorption cross section is

$$
C_{a}^{e}=\frac{\omega}{c} V_{n p} \varepsilon_{h}^{3 / 2}\left|\frac{3 \varepsilon}{\varepsilon+2 \varepsilon_{h}}\right|^{2} \operatorname{Im} \frac{-1}{\varepsilon_{e f f}},
$$

where $V_{n p}=4 \pi R^{3} / 3$ is the nanoparticle volume and where all permittivities are relative. The $d_{r}$ correction is not present for the magnetic dipole contribution since that is governed by the tangential electric fields, and it has been shown that for the magnetic dipole contribution the effect of $d_{\theta}$ is very small, ${ }^{23}$ so that we just apply the surface corrections to the electric dipole contribution.

Since $d_{\theta} / \varepsilon R$ is small, we can make the expansion

$$
\frac{-1}{\varepsilon_{\text {eff }}} \simeq \frac{-1}{\varepsilon}+\left(\frac{1}{\varepsilon}-\frac{1}{\varepsilon_{h}}\right) \frac{d_{r}}{R}+\frac{2}{\varepsilon^{2}} \frac{d_{\theta}}{R} .
$$

The first term in this equation gives the classical volume contribution to the absorption cross section (i.e., $C_{a}^{e}$ in Sec. II), the second gives the electronic spill-out surface contribution and the third gives the surface contribution due to electronic surface scattering. Since we are interested in the low frequency regime the particle conductivity is very large and the uniform interior field is very small in the particle bulk. Therefore, we expect the surface contributions to be more important than the volume one.

Next, we estimate each contribution separately, which is a novel analysis for low frequencies. We take the limits $\omega \ll \omega_{p}$ and $\omega \tau_{0} \ll 1$ in the bulk dielectric function. Then, the classical volume effect is

$$
\operatorname{Im} \frac{-1}{\varepsilon} \simeq \frac{\omega}{\omega_{p}} \frac{1}{\omega_{p} \tau_{0}} .
$$

With respect to the surface contributions, we also approximate $d_{r}$ and $d_{\theta}$ by their corresponding values for a planar surface. This is justified because these lengths are of the order of a few Ångström while particle radii are much larger than this. The surface contribution in Eq. (12) due to excitation of surface electron-hole pairs is ${ }^{20,24}$

$$
\operatorname{Im}\left[\left(\frac{1}{\varepsilon}-\frac{1}{\varepsilon_{h}}\right) \frac{d_{r}}{R}\right] \simeq \frac{1}{\varepsilon_{h}} \operatorname{Im} \frac{-d_{r}}{R} \simeq \frac{1}{6 \varepsilon_{h}} \frac{\hbar \omega}{E_{F}} \eta\left(r_{s}\right) \frac{1}{k_{F} R}
$$

where $E_{F}$ and $k_{F}$ are the Fermi energy and the Fermi wave vector, respectively, and $\eta$ is a number depending on the metal via its one-electron radius $r_{s}$. $\eta$ has been estimated to be of the order of 1 in Ref. 24 .

Finally, in order to estimate the surface contribution to the absorption cross section due to electronic surface scattering, we will adopt the simple model of Ref. 22 in which the electron scattering is determined by a phenomenological roughness parameter $p, 0 \leq p \leq 1$. A very rough inhomogeneous surface is described by $p=0$, while $p=1$ for a perfectly reflecting surface. The use of the semiclassical infinite barrier model for the metal/medium interface also allows for a simple analytical evaluation of $d_{\theta}$, as expressed by Eq. (12) in Ref. 22. In the low frequency limit, this yields,

$$
\operatorname{Im}\left(\frac{2}{\varepsilon^{2}} \frac{d_{\theta}}{R}\right) \simeq \frac{1-p}{\sqrt{10}} \frac{\left(\omega \tau_{0}\right)^{1 / 2}}{\omega_{p} \tau_{0}} \frac{v_{F}}{\omega_{p} R} .
$$

Note that the absorption cross section goes to zero as $\omega$ goes to zero. However, the contribution due to the microscopic surface roughness to $1 / \varepsilon_{\text {eff }}$ shows a slower decrease with $\omega$, going as $\omega^{1 / 2}$ instead of $\omega$.

Putting all the contributions together, we can finally write the effective dielectric function as

$$
\varepsilon_{e f f} \simeq i \frac{\omega_{p}^{2} \tau_{e f f}}{\omega}
$$

where $1 / \tau_{\text {eff }}$ is given by

$$
\frac{1}{\tau_{\text {eff }}}=\frac{1}{\tau_{0}}+\frac{1}{6 \varepsilon_{h}} \frac{\hbar \omega_{p}}{E_{F}} \eta\left(r_{s}\right) \frac{\omega_{p}}{k_{F} R}+\frac{1-p}{\sqrt{10}} \frac{1}{\left(\omega \tau_{0}\right)^{1 / 2}} \frac{v_{F}}{R} .
$$

We can use this effective dielectric function to calculate the electric dipolar part of the absorption cross section for coated 
or uncoated spheres taking into account surface effects. In writing Eq. (16), we have taken into account that the real part of $\varepsilon_{\text {eff }}$ is going to be unimportant since its imaginary part will dominate at small frequencies. Note that the last term in Eq. (17) is $\omega$-dependent and can dominate the value of $\tau_{\text {eff }}$ at sufficiently low frequencies, independent of particle size.

To reconsider the previous example of a gold $R=20$ nm sphere in water at $f=13 \mathrm{MHz}$, using $p=0$ we obtain $\tau_{\text {eff }}=5.8 \times 10^{-17} \mathrm{~s}$, a more than two order of magnitude decrease from the bulk value (most of which is contributed by $d_{\theta}$; for $p=1, \tau_{\text {eff }}=1.5 \times 10^{-14} \mathrm{~s}$ ). The resulting absorption cross section is dominated by the electric dipole contribution $^{25}$ and at $13 \mathrm{MHz}$ exhibits an increase of nearly two orders of magnitude compared to the values obtained using $\tau_{0}$, as shown in Fig. 1 (solid line indicated by $\tau_{\text {eff }}$ ). However, if we add the same coating as above, the absorption cross section is unaffected by the value of the relaxation time (dashed line indicated by $\tau_{\text {eff }}$ ) since most absorption occurs in the coating.

Changes in $\tau$ due to grain boundary scattering may also be relevant for bare nanoparticles, but, again, an absorptive coating (or absorptive host) will render any such changes to $\tau$ unimportant. Another possible mechanism is enhanced absorption through the excitation of surface phonons. However, as shown in Ref ${ }^{26}$ even at $1.7 \mathrm{THz}$ the phonon contribution is smaller than the electron-hole pair surface contribution, and the phonon contribution decays rapidly with lower frequencies. Finally, we want to mention that there have been several attempts in the literature to include surface roughness in the optical response, see e.g. Ref. 27 and $^{28}$ However, since we have no clear information about the surface morphology, their relevance for the present work cannot be clearly judged.

\section{THERMODYNAMICAL ANALYSIS}

In the following we show that absorption by non-interacting spherical metal nanoparticles cannot cause the heating observed in the reported experiments. To do this, we solve the equation for the flow of heat in a medium characterized by its thermal conductivity, specific heat $c_{v}$, and mass density $\rho$. We assume that the heat source is a collection of $N_{p}$ point sources (representing the nanoparticles) placed at positions $R_{p}$. The heat equation is

$$
\kappa \nabla^{2} T(\mathbf{r}, t)-\rho c_{v} \frac{\partial T}{\partial t}+Q(\mathbf{r}, t)=0
$$

the expression for the source $Q\left(\mathrm{~W} / \mathrm{m}^{3}\right)$ being

$$
Q(\mathbf{r}, t)=\theta(t) Q_{0} \sum_{\mathbf{R}_{\mathbf{p}}} \delta\left(\mathbf{r}-\mathbf{R}_{\mathbf{p}}\right)
$$

where $\theta(t)$ is the Heaviside function. Here, $Q_{0}$ is the energy absorbed per unit time by one nanoparticle. We are implicitly assuming that the absorbed EM energy is efficiently converted to heat. ${ }^{29}$ Equations. (18) and (19) can be solved by standard methods considering an infinite medium, ${ }^{30}$ giving the rate of increase in temperature as

$$
\frac{d T(\mathbf{r}, t)}{d t}=Q_{0} \frac{\sqrt{\pi \rho c_{v}}}{8 \pi^{2}(\kappa t)^{3 / 2}} \sum_{R_{p}} \exp \left[-\frac{\left|\mathbf{r}-\mathbf{R}_{\mathbf{p}}\right|^{2}}{4 \frac{\kappa t}{\rho c_{v}}}\right] .
$$

From Eq. (20) the temperature $T(\mathbf{r}, t)$ is obtained by integration. The temperature thus obtained is consistent with Eq. (2) of Ref. 3.

Since we have $N_{p}$ terms in the sum appearing in Eq. (20), we divide this sum by $N_{p}$ and, correspondingly, multiply $Q_{0}$ by $N_{p}$. Then we compute $d T(\mathbf{r}, t) / d t$ for a constant value of $N_{p} Q_{0}=1 \mathrm{~W}$. This allows us to plot results for different numbers of particles on the same scale. For simplicity, we choose a system with cubic symmetry and put the $N_{p}$ particles in a cubic volume $L^{3}$. In Fig. $2, L=1 \mathrm{~cm}(1 \mathrm{~mL}$ volume), corresponding to the approximate volume of the experimental cuvette in Ref. 7. We use parameters appropriate for water: $c_{v}=4.18 \times 10^{3} \mathrm{~J} / \mathrm{kg}-\mathrm{K}, \rho=1000 \mathrm{~kg} / \mathrm{m}^{3}$, $\kappa=0.61 \mathrm{~W} / \mathrm{m}-\mathrm{K}$.

Results are shown for $N_{p}=100^{3}$ (solid lines) and $N_{p}=500^{3}$ (dashed lines) particles and at different points $r=(x, y, z), x=y=z, \mathrm{~nm}$ (top curve), $x=1 \mathrm{~mm}$ (middle curve), and $x=2.5 \mathrm{~mm}$ (lower curve) with the origin placed at the center of the cubic cuvette. We see that the temperature is rather uniform over the cuvette and is essentially independent of the number of particles. Note also that one needs $N_{p} Q_{0} \sim 1 \mathrm{~W}$ to obtain significant heating. It is easy to see that this level of heating is impossible to achieve for metal nanoparticles at RF-THz frequencies. For example, assume the same nanoparticles as in the previous examples (gold, $R=20 \mathrm{~nm}$ ) at $13 \mathrm{MHz}$. Consider a spherical cuvette of radius $a=7.1 \mathrm{~mm}(1.5 \mathrm{~mL}$ volume $)$ in air and containing a deionized water and nanoparticle solution. Assume a field of 15 $\mathrm{kV} / \mathrm{m}$ in air, the maximum field considered in, e.g., Ref. 7. The electric field inside the cuvette is $550 \mathrm{~V} / \mathrm{m}$, and the intensity incident on each nanoparticle is $I=3.6 \times 10^{3} \mathrm{~W} / \mathrm{m}^{2}$. Then, $Q_{0}^{\text {bare }}=C_{a}^{\text {bare }} I \simeq 1.6 \times 10^{-24} \mathrm{~W}$ and $Q_{0}^{\text {coated }}=C_{a}^{\text {coated }}$ $I \simeq 1 \times 10^{-19} \mathrm{~W}$. Even assuming a volume fraction of one $\left(N_{p} \sim 10^{16}\right)$ we would have for the coated sphere $N_{p} Q_{0}^{\text {coated }} \sim 0.001 \mathrm{~W}$, which is insufficient for heating. For

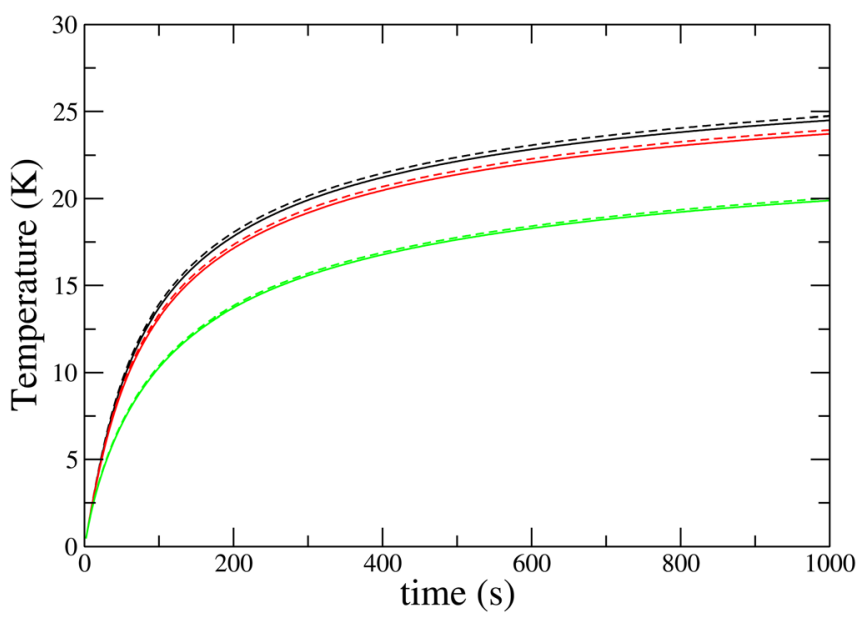

FIG. 2. (Color online) Temperature vs time at different points $r=(x, y, z)$, $\mathrm{x}=y=\mathrm{z}$, with the origin at the center of a cube of side $L=1 \mathrm{~cm}$ for $N_{p}=100^{3}$ (solid lines) and $N_{p}=500^{3}$ (dashed lines). $x=10 \mathrm{~nm}$ (top curve), $x=1 \mathrm{~mm}$ (middle curve), and $x=2.5 \mathrm{~mm}$ (lower curve). 
the small volume fractions used in the RF experiments $\left(\sim 10^{-6}\right)$, or for any other reasonable volume fraction that may be considered, no RF heating should be observed, consistent with the findings of Ref. 13. The same can be said for $\mathrm{GHz}$ frequencies. At, e.g., $1 \mathrm{GHz}, C_{a}^{\text {bare }} I \simeq 1.3 \times 10^{-21} \mathrm{~W}$ and $C_{a}^{\text {coated }} I \simeq 1 \times 10^{-19} \mathrm{~W}$.

Further conclusions can be drawn from the steady state analysis. Assuming non-interacting, non-aggregated nanoparticles, the steady state temperature at the surface of a spherical cuvette is ${ }^{17}$

$$
\Delta T=\frac{N_{p} Q_{0}}{4 \pi a \kappa_{m}}
$$

where $a$ is the radius of the cuvette and $\kappa_{m}$ is the thermal conductivity of the medium outside of the cuvette. The associated heating rate (degrees/s) is, $H R=N_{p} Q_{0} \rho c_{v} V$, where $V$ is the volume of the cuvette. Assuming a $1.5 \mathrm{~mL}$ volume and thermal conductivity same as water, the necessary power (W) to increase the temperature $\Delta T$ is $N_{p} Q_{0}=4 \pi a \kappa_{m} \Delta T=0.055 \Delta T$. For $\Delta T=20$ degrees as in Fig. $2, N_{p} Q_{0}=1.1 \mathrm{~W}$, in good agreement with the transient analysis. The heating rate is $H R=0.16^{\circ} / \mathrm{s}$, also in good agreement with the linear part of the heating curves. If the cuvette resides in air, the low thermal conductivity of the air results in $N_{p} Q_{0}=0.05 \mathrm{~W}$ needed to reach $\Delta T=20$ degrees. Expressed in terms of volume fraction $\mathrm{F}_{v}$, the required value of $C_{a} I$ to yield a $\Delta T$ temperature increase is, according to Eq. (21),

$$
C_{a} I=\frac{4 \pi \kappa_{m} R^{3} \Delta T}{F_{v} a^{2}} .
$$

For $R=20 \mathrm{~nm}$ and considering a $1.5 \mathrm{~mL}$ spherical cuvette in air, for a volume fraction of $10^{-6}$ we would need $C_{a} I$ order $10^{-12} \mathrm{~W}$ (i.e., for each nanoparticle) to achieve $\Delta T$ in the tens of degrees. Assuming electric fields in the $\mathrm{kV} / \mathrm{m}$ range outside the cuvette, this order of absorption cross section cannot be achieved at RF, where $C_{a} I$ is $10^{-19} \mathrm{~W}$ per nanoparticle in the example above.

The conclusions above pertain to nanoparticles in a nonabsorbing host medium. It is worthwhile to see if nanoparticles can contribute to heating in an absorbing host medium, or if the host medium absorption itself is the dominant contribution. In Ref. 13, the observed RF heating was completely attributed to Joule heating of the ionic host medium. The authors also argue that this mechanism is likely responsible for the heating observed in Refs. $31-33$ in the $1-12$ $\mathrm{GHz}$ range.

\section{ABSORBING HOST MEDIUM}

To investigate an absorbing host medium we consider nanoparticles in liquids characterized by the Debye (ColeCole) form

$$
\varepsilon_{r}(\omega)=\varepsilon_{r}(\infty)+\frac{\varepsilon_{r}(0)-\varepsilon_{r}(\infty)}{1-\left(i \omega \tau_{w}\right)^{1-\alpha}}+i \frac{\sigma}{\omega \varepsilon_{0}} .
$$

We consider pure water (experimentally, this would be deionized water) at $25^{\circ} \mathrm{C}$, where $\varepsilon_{r}(0)=78.36, \varepsilon_{r}(\infty)=5.2$, $\alpha=0.02, \sigma=0$, and $\tau_{w}=8.27$ ps (Ref. 34) $\left(1 / 2 \pi \tau_{w}=19\right.$ $\mathrm{GHz})$, and physiological saline, where $\varepsilon_{r}(0)=76$, $\varepsilon_{r}(\infty)=4.5, \alpha \simeq 0.02, \sigma=1.45 \mathrm{~S} / \mathrm{m}$, and $\tau=8.11$ ps. Since Mie theory is only applicable to a nonabsorbing host medium, we compute the effective permittivity $\varepsilon_{e f f}$ of the nanoparticle-liquid solution using the Maxwell-Garnett mixing formula. ${ }^{35}$ From this, we compute the absorption cross section of a $1.5 \mathrm{~mL}$ cuvette containing pure water or saline solution in air. Figure 3 shows the absorption cross section normalized by cross-sectional area of the cuvette $\left(A=1.58 \times 10^{-4} \mathrm{~m}^{2}\right)$ for a moderately-large nanoparticle volume fraction of 0.01 , for three different values of nanoparticle conductivity, $\sigma_{\text {part }}=0,0.1$ and $10^{7} \mathrm{~S} / \mathrm{m}$, and for unity nanoparticle permittivity. For the saline host medium, absorption is clearly independent of the presence or absence of the nanoparticles, regardless of nanoparticle conductivity (the three curves are indistinguishable on the scale of the plot). This indicates that spherical nanoparticles cannot enhance absorption in saline-like fluids in the considered frequency range. For the water host, below approximately 100 $\mathrm{MHz}$ water absorption is small enough so that nanoparticles with an appropriate value of conductivity can enhance absorption. From Ref. 17, $\sigma_{\text {part }}=0.1 \mathrm{~S} / \mathrm{m}$ approximately maximizes absorption in the low $\mathrm{MHz}$ range, hence the enhanced absorption shown by the long-dashed curve in Fig. 3. At these lower frequencies the absorption of metallic nanoparticles $\left(\sigma=10^{7} \mathrm{~S} / \mathrm{m}\right)$ is too small, even at the high volume fraction considered, to enhance absorption compared to that of the water. In fact, replacing the metallic nanoparticles with air voids having $\sigma=0 \mathrm{~S} / \mathrm{m}$ (solid line) leads to the same net absorption. Above approximately $100 \mathrm{MHz}$ the absorption of the nanoparticle-water solution is independent of the presence or absence of nanoparticles of any material composition. Therefore, in all cases involving metal nanoparticles, absorption and subsequent heating is due to the absorbing host medium, and is not influenced by the presence or absence of spherical nanoparticles. However, needlelike objects such as carbon nanotubes or elongated ellipsoidal particles maintain a strong radial near-field in the vicinity

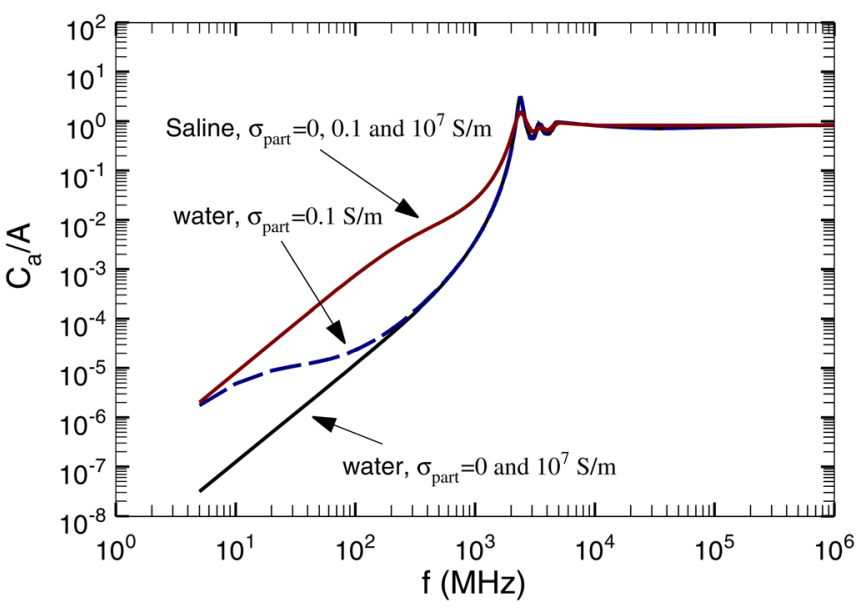

FIG. 3. (Color online) Absorption cross section of a $1.5 \mathrm{~mL}$ spherical cuvette in air, normalized by cross-sectional area of the cuvette, for a nanoparticle volume fraction of 0.01 and for three different values of nanoparticle conductivity $\left(\sigma=0,0.1\right.$ and $\left.10^{7} \mathrm{~S} / \mathrm{m}\right)$. 
of the tube ends (e.g., lightning-rod effect) that can significantly enhance absorption in a conducting host. ${ }^{36}$

Finally, it should be noted that nanoparticle agglomeration has not been considered here, and could possibly contribute to increased heating.

In conclusion, we have analyzed several mechanisms by which spherical nanospheres can absorb RF-far infrared radiation which can be subsequently released to their host medium as heat. Considering volume and surface effects we determine absorption cross sections. By performing a thermodynamical analysis we find that these values of cross sections at the incident power levels typical of experiments are unable to produce the increase in temperature of tens of degrees reported in many RF-GHz experiments. Our analysis also confirms recent experimental results showing that the Joule heating of the solution containing the nanoparticles is the mechanism producing such rises of temperatures, and that the presence of nonaggregated metallic nanoparticles plays no role in heating. We confirm that Joule heating of the host medium (including pure water) should also be the heating mechanism operating in the microwave range of the EM spectrum.

\section{ACKNOWLEDGMENTS}

Support by the Spanish Ministerio de Ciencia e Innovación Grant No. FIS2008-04209 and the Swedish Foundation for Strategic Research (metamaterial Grant No. SSF RMA08-0109) is acknowledged.

${ }^{1}$ L. C. Kennedy, L. R. Bickford, N. A. Lewinski, A. J. Coughlin, Y. Hu, E. S. Day, J. L. West, and R. A. Drezek, Small 7, 169 (2011).

${ }^{2}$ P. M. Bendix, S. N. S. Reihani, and L. B. Oddershede, ACS Nano 4, 2256 (2010).

${ }^{3}$ P. Keblinski, D. G. Cahill, A. Bodapati, C. R. Sullivan and T. A. Taton, J. Appl. Phys. 100, 054305 (2006).

${ }^{4}$ S. A. Curley, P. Cherukuri, K. Briggs, C. R. Patra, M. Upton, E. Dolson, and P. Mukherjee, J. Exp. Ther. Oncol.7, 313 (2008).

${ }^{5}$ C. J. Gannon, C. R. Patra, R. Bhattacharya, P. Mukherjee, and S. A Curley, Nanobiotechnology 6, 2 (2008).

${ }^{6}$ J. Cardinal, J. Klune, E. Chory, G. Jeyabalan, J. Kanzius, M. Nalesnik, and D. Geller, Surgery 144, 125 (2008).

${ }^{7}$ C. H. Moran, S. M. Weinerdi, T. K. Cherukuri, C. Kittrell, B. J. Wiley, N. W. Nicholas, S. A. Curley, J. S. Kanzius, and P. Cherukuri, Nano Res. 2, 400 (2009).

${ }^{8}$ P. Cherukuri, E. S. Glazera, and S. A. Curley, Advanced Drug Delivery Rev. 62, 339 (2010).
${ }^{9}$ P. Cherukuri and S. A. Curley, Cancer Nanotechnology, Methods in Molecular Biology 624, 359 (2010).

${ }^{10}$ E. S. Glazer, C. Zhu, K. L. Massey, C. S. Thompson, W. D. Kaluarachchi, A. N. Hamir, and S. A. Curley, Clin. Cancer Res. 16, 5712 (2010).

${ }^{11}$ E. S. Glazer, K. L. Massey, C. Zhu, and S. A. Curley, Surgery 148, 319 (2010).

${ }^{12}$ E. S. Glazer, C. Zhu, K. L. Massey, C. S. Thompson, W. D. Kaluarachchi, A. N. Hamir, and S. A. Curley, Clin. Cancer Res. 16, 5712 (2010).

${ }^{13}$ D. Li, Y. S. Jung, S. Tan, H. K. Kim, E. Chory, and D. A. Geller, J. Colloid Interface Sci. 358, 47 (2011).

${ }^{14}$ C. F. Bohen and D. R. Huffman, Absorption and Scattering of Light by Small Particles (Wiley, Weinheim, 1983); J. A. Stratton, Electromagnetic Theory (McGraw-Hill, New York, 1941).

${ }^{15}$ P. N. Sen and D. B. Tanner, Phys. Rev. B 26, 3582 (1982).

${ }^{16}$ R. P. Devaty and A. J. Sievers, Phys. Rev. B 41, 7421 (1990).

${ }^{17}$ G. W. Hanson and S. K. Patch, J. Appl. Phys. 106, 054309 (2009).

${ }^{18}$ Sigma-Aldrich Corporation, see http://www.sigmaaldrich.com.

${ }^{19}$ P. de Andrés, R. Monreal, and F. Flores, Phys. Rev. B 32, 7878 (1985).

${ }^{20}$ P. Apell and D. R. Penn, Phys. Rev. Lett. 50, 1316 (1983).

${ }^{21}$ P. Apell and Å. Ljungbert, Physica Scr. 26, 113 (1982).

${ }^{22}$ P. Apell, R. Monreal, and F. Flores, Solid State Commun. 52, 971 (1984).

${ }^{23}$ P. Apell, Physica Scr. 29, 146 (1984).

${ }^{24}$ B. N. J. Persson and P. Apell, Phys. Rev. B 27, 6058 (1983).

${ }^{25}$ Using $\tau_{0}$, the electric dipole contribution is an order of magnitude smaller than the magnetic dipole term, but when using $\tau_{\text {eff }}$ in the electric dipole term and $\tau_{0}$ in the magnetic dipole term the roles are reversed and the electric dipole term becomes an order of magnitude larger than the magnetic contribution.

${ }^{26}$ R. Monreal, J. Giraldo, F. Flores, and P. Apell, Solid State Commun. 54, 661 (1985).

${ }^{27}$ H. J. Trodhal, Journal of Physics C- Solid Stat. Phys. 15, 7245 (1982).

${ }^{28}$ E. J. Austin and M. Wilkinson, J. Phys.:Condens. Matter 5, 8461 (1993).

${ }^{29}$ H. H. Richardson, M. T. Carlson, P. J. Tandler, P. H., and A. O. Govorov, Nano Lett. 9, 1139 (2009).

${ }^{30}$ J. Mathews and R. L. Walker, Mathematical Methods of Physics (Addison-Wesley, Reading, MA 1970).

${ }^{31}$ K. Hamad-Schifferil, J. J. Schwartz, A. T. Santos, S. Zhang, and J. M. Jacobson, Nature 415, 152 (2002).

${ }^{32}$ M. J. Kogan, N. G. Bastus, R. Amigo, D. Grillo-Bosch, E. Araya, A. Turiel, A. Labarta, E. Giralt, and V. F. Puntes, Nano Lett. 6, 110 (2006).

${ }^{33}$ K. Aslan and C. D. Geddes, Anal. Chem.79, 2131 (2007).

${ }^{34}$ U. Kaatze, J. Chem. Eng. Data 34, 371 (1989).

${ }^{35}$ Here, we use the Maxwell-Garnett (MG) effective permittivity. In a nonabsorbing host medium for large particle conductivity, the magnetic dipole contribution can dominate absorption, which is not accounted for in the MG effective permittivity. Hence, one can question the applicability of this method for the $\sigma=10^{7} \mathrm{~S} / \mathrm{m}$ case in Fig. 3. However, it is easy to show using the polarizability of such a nanoparticle with a water or saline coating that the absorption in the coating renders the magnetic dipole contribution negligible, even for low frequencies where the water host is weakly-absorbing, and so the results in Fig. 3 for the metal nanoparticle are valid.

${ }^{36}$ M. V. Shuba, G. Ya. Slepyan, S. A. Maksimenko, and G. W. Hanson, J. Appl. Phys. 108, 114302 (2010). 